\title{
A situação da pesca na Amazônia Central
}

\author{
Ulrich Saint-Paul * \\ Peter B. Bayley *
}

\section{ASPECTOS HIDROLÓGICOS}

O extenso sistema fluvial da Amazônia, a maior área hidrogeográfica do mundo, sempre teve grande importância como fonte de alimentação, bem como meio de transporte, cuja influência na ecologia e climatologia desta região é considerável.

O rio Solimões/Amazonas com seus tributários drena uma área de aproximadamente 6,5 milhões de $\mathrm{km}^{2}$ (incluindo a área do rio Tocantins) (Haffer, 1974) e joga no oceano Atlântico em média uma quantidade de água de 218.000 $\mathrm{m}^{3} / \mathrm{s}$, isso é, $15-20 \%$ de toda água doce lançada no mar (Sioli, 1967).

Sioli (1950 e 1967) classificou os três tipos de água deste sistema fluvial, em água branca, água preta e água clara. Elas dependem da situação geológica nas regiões das cabeceiras e são caracterizadas por diferentes parâmetros químicos e físicos.

A produtividade biológica, conseqüentemente a produção pesqueira, não é igıial em todos tipos de água, mas depende da situação limnológica. Portanto, as águas pretas e até certo ponto as águas claras, podem ser classificadas como águas de baixo potencial pesqueiro. Em contrapartida as águas brancas são consideradas as mais produtivas e conseqüentemente têm una aita produção pesqueira (Geisler et al., 1971; Junk \& Honda, 1976).

Considerando este fato, o potencial pesqueiro limita-se ao rio Solimões/Amazonas com seus tributários principais como p.ex. o Japurá, Purus, Juruá ou Madeira.

\section{ECOLOGIA E SITUAÇÃO DA PESCA}

Na Amazônia, desenvolve-se uma ictiofauna mais rica que nos outros sistemas fluviais do mundo. Até o ano 1967, já foram descritas 1.300 espécies endêmicas de peixes (Roberts, 1972). O número total porém deve atingir mais do que 2.000 espécies (Geisler et al., 1971). Das espécies descritas, $85 \%$ são Ostariophysi, incluindo Characidae $(43 \%)$, Siluriformes
(39\%) e Gymnotidae (3\%). O resto pertence às famílias dos Lepidosirenae, Osteoglossidae, Nandidae, Cichlidae, Cyprinodontidae, Poeciliidae, Galaxiidae e Percichthydae (Roberts 1972) .

Os níveis das águas, nos rios amazónicos, esião submetidos a grandes oscilações, as quais modificam substancialmente as situações ecológıcas e, como ainda será mostrado, conseqüentemente as condições de captura de peixes. No rio Solimões, são medidas flutuações anuais entre 16 e $20 \mathrm{~m}$, no rio Negro, em Manaus, de 10 a $16 \mathrm{~m}$, e no baixo Amázonas ainda de 5 a $7 \mathrm{~m}$ (Wiihelmy, 1970). O nível máximo em Manaus é atingido em junho, descendo depois de alguns dias e atingindo o nível minimo no mês de novembro. Assim, ocorrem anualmente grandes inundações que formam as paisagens da várzea e da floresta inundada.

As várzeas são chamadas as paisagens de inundação ao longo do curso dos rios de água branca. Durante a enchente, os lagos ficam cheios com água branca. A produtiviaade destas áreas depende desta inundação. Depois de uma fase de sedimentação do material em suspensão da água branca, ocorre uma alta produção primária (Schmidt, 1973). Nesta época, desenvolvem-se também, as macrófitas aquáticas que formam uma extensa vegetação flutuante. Junk (1970 e 1973a), realizou estudos minuciosos sobre a ecologia deste biótopo mostrando também sua importância para a ictiofauna. Assim, permanece durante a cheia uma extensa área em disponibilidade para o povoameno pela fauna aquática, que na época da vazante diminui (Junk, 1973 b). Muitas espécies de peixes começam a entrar na várzea aproveitando a alta quantidade de perizoon nas raízes das macrófitas aquáticas e suas sementes como fonte de alimentação (Junk, 1973 a; 1975). Por exemplo, Astronotus ocellatus e outras espécies da família de Cichlidae aiimentam-se dos invertebrados da zona radicular das macrófitas e Colossoma macropomum das se-

* Instituto Nacional de Pesquisas da Amazônia, Manaus - AM 
mentes de Oryza perennis no início da época de cheia.

Muitas espécies que entram na várzea não ficam nos próprios lagos, mas migram para a floresta inundada da várzea. Esta floresta tem uma flora característica, bem adaptada às oscilações da água. Na época da cheia, estas árvores começam a produzir frutos e sementes que servem como fonte alimentar para os peixes frutívoros como p.ex. Colossoma macropomum, Colossoma brachypomum, Brycon sp., Mylossoma sp. ou Triportheus sp. (Goulding, 1979) .

Knöppel (1970) mostrou, pela primeira vez, a importância das substâncias nutritivas alóctones nas cadeias alimentares de algumas espécies de peixes nos igarapés de terra firme. Tráta-se com preferência de frutas, pólens, larvas de insetos, insetos, etc.. Estas investigaçōes foram realizadas com peixes de água clara e de água preta, mas valem também para biótopos da floresta inundada com uma baixa produção autóctone.

Com a queda do nível da água, termina tanibém o período produtor das árvores da floresta inundeda e da vegetação flutuante da várzea. A maioria dos lagos seca e os peixes são obrigados a sair desta área. Muitas espécies como Colossoma macropomum, Colosso. ma brachypomum ou Brycon sp. migram para os grandes rios e passam lá um periodo de jejum que pode durar alguns meses. Entretanto, eles armazenam muita gordura no corpo que os faz sobreviver esta fase sem prejuízos (Goulding, 1979). As espécies não migratórias que ficam nos lagos durante esta época, têm que ser bem adaptadas para sobreviverem em condições hipóxias. Assim Arapaima gigas, Electrophorus electricus, Acanthicus histrix e outros respiram ar atmosférico. Outras espécies como p.ex. Colossoma macropomum apresentam uma forma de adaptação que permite sua sobrevivência por algumas horas em águia com uma concentração de oxigênio abaixo de $1 \mathrm{ppm}$, muito embora este sistema adaptador seja ainda desconhecido.

Estas flutuações normais da água não provocam somente uma mudança de hábitos ali- mentares em muitas espécies, mas também migrações de peixes. Isso acontece com a maioria das espécies de Characidae e das espécies maiores do grupo de Siluriformes que saem para os rios e ficam nas "pausadas" ( $\left.{ }^{1}\right)$ ate à desova. Mudanças no nível da água, após as primeiras grandes precipitações e chuvas contínuas, causam variações na condutipilidade e pH, tendo importância vital para o desenvolvimento gonadal e na provocação da desova como foi mcstrado por Kirschbaum (1979) para a espécie Eigenmannia virescens (Rhamphichthyidae).

Depois da incubação os alevinos dos caracoidae migradores, migram para a várzea inundada, onde são encontrados em grandes quantidades. Eles aproveitam a melhor oferta de alimentação associada à vegetação flutuante. Os adultos também voltam dos afluentes depois da desova e entram nos lagos procurando alimentação.

Estas grandes oscilações no nível de água dos rios modificam também substancialmente as condições de captura (McConnell, 1975; Petrere, 1978). Há uma maior oferta de pescado, no início da vazante, em agosto e setembro. No período da cheia, nos meses de abril e maio, ocorre exatamente o contrário. Petrere (1978) calculou para o ano 1976 , um total de $30.800 \mathrm{t}$ de pescado desembarcado pela frota pesqueira de Manaus. A captura mínima $(4,3 \%$ da captura total) aconteceu no mês de abril e a máxima $(13,0 \%)$ no mês de setembro. Assim, ocorre todo ano na época da cheia, uma queda acentuada no abastecimento por alguns meses, que provoca um aumento rápido nos preços do pescado, atingindo muitas vezes até $250 \%$ (Honda et al., 1975). Goulding (1979) descobriu a mesma sazonalidade nas capturas da frota pesqueira de Porto Velho em relação ao nível da água.

Este fenômeno pode ser ainda explicado como uma super-exploração dos cardumes de peixes durante as migrações de piracema. Especialmente o Colossoma macropomum é explorado nesta época mas também Brycon sp., Semaprochilodus sp., Colosscma brachypomum e outros.

(1) - Expressão regional para áreas que possuem madeiras acumuladas. 
Petrere (1978) revelou que somente 31 espécies têm importância como peixes comerciais e são desembarcados no mercaúo de Manaus. $44,1 \%$ do pescado totai desembarcado em 1976, foi de Colossoma macropomum e $16,0 \%$ de Semaprochilodus sp. No mercado de Porio Velho, também não chegaram mais do que 30 diferentes espécies em 1977. Brycon sp. representou $23 \%$ da produção total (Goulding, 1979). Smith (1979) verificou que, na área da Itacoatiara, estão sendo consumidas aproximadamente 86 diferentes espécies de peixes dos quais $62 \%$ são Semaprochilodus sp.

A Tabela 1 mostra um resumo das espécies mais freqüentes desembarcadas em Manaus (1976), Porto Velho (1977) e Itacoatiara (1977).

O produto total da pesca da bacia do alto e médio Amazonas foi em 1977 de aproximadamente 85.300 t (Bayley, 1978). Em geral, pode constatar-se que o esforço de pesca aumentou muito nos últimos anos. Como alguns estoques pesqueiros já são bastante explorados nas proximidades de Manaus, os pescadores são obrigados a realizar viagens para lugares de captura mais distantes (Petrere, 1978). Já são feitas viagens de pesca de até três meses. Para o rio Madeira foi observado o mesmo fato (Goulding, 1979).

Como os preços de óleo Diesel e gelo aumentaram drasticamente nos últimos ànos, essas viagens só compensam o investimento quando são capturados peixes de um alto valoi comercial, como p.ex. Colossoma macropomum ou Arapaima gigas. Conseqüentemente, acontece uma captura especializada destas espécies. Por outro lado, estas despesas têm sido o maior fator para a redução das viagens pesqueiras, as quais diminuíram de $6.501 \mathrm{em}$ 1976 para 5.484 em 1977 na frota pesqueira de Manaus. Esta baixa de $16 \%$ foi possivelmente a causa principal na redução de $20 \%$ da captura total (Bayley, 1978) .

Aplicando os dados teóricos de Weicomme (1976) sobre o rendimento da pesca nos grandes rios inundados da África para o riu Solimões/Amazonas, Bayley (1978) estimou uma possível duplicação do rendimento total neste sistema fluvial. Isso não significaria uma exploração mais intensiva dos estoques pesqueiros explorados, mas uma mudança da composi- ção qualitativa dos pescados desembarcados. Esta pesca poderia ser baseada em espécies de tamanho médio da várzea como Semaprochilodus sp., Prochilodus sp., Triportheus sp. e outras espécies das famílias Curimatidae, Anostomidae e Hemidontidae.

\section{PEIXES COMO ALIMENTO DA POPULAÇÃO}

Tradicionalmente, o peixe é uma das fontes de proteina mais importante na Amazônia. Uma analise feita por Shrimpton et al. (1979) de um levantamento do Governo do Amazonas sobre os alimentos consumidos por 1.200 famílias em Manaus durante os anos 1973/74 (CODEAMA, 1977) revelou que cerca de $64,4 \%$ da proteína consumida foi de peixe. O consumo de peixe na classe média com 2,2 a 5,2 unidades salariais mínimas fica perto de $139 \mathrm{~g} /$ pessoa / dia. Na classe com um salário abaixo de 2,2 unidades salariais mínimas o consumo de peixe é ainda mais alto: $151 \mathrm{~g} / \mathrm{pessoa} / \mathrm{dia}$. Com o crescimento permanente da população da Amazônia e especialmente da capital, Manaus, aumentará a demanda do pescado. $\mathrm{Na}$ área de Itacoatiara, o consumo de peixe é de $104 \mathrm{~g} / \mathrm{pessoa} / \mathrm{dia}$, mas com grandes variações sazonais (Smith, 1979) . Na época da seca a oferta e consumo do pescado é maior do que na época da cheia. Para o território de Rondônia, Bayley (1978) calculou um consumo de peixe bem mais baixo: em 1977, foi de apenas $27 \mathrm{~g} /$ pessoa/dia.

\section{PPOBLEMAS DO ABASTECIMENTO DA POPULAÇÃO RURAL COM PEIXE}

Um dos maiores problemas na colonização da Amazôniá é a abastecimento dos colonos com proteína animal. Um levantarnento de Goulding (1979) mostrou que em Roraina. as zonas rurais e dos pequenos centros urbanos, são abastecidas com peixes capturados nas proximidades. Esta observação se faz também no resto da Amazônia. A maioria dos projetos de colonização é realizado nas margens das rodovias como a Transamazônica, as estradas Manaus-Boa Vista ou Manaus-Itacoatiara. Todas estas estradas atravessam áreas com igarapés pobres em nutrientes os quais, em condições naturais côntêm conseqüentemente poucos peixes (Geisler et al., 1971; Junk, 1975). Como o abastecimento destas colô- 
TABELA 1 - As dez espécies de peixes mais freqüentes desembarcadas em Manaus (Petrere, 1978), Porto Velho (Goulding, 1979) e ltacoatiara (Smith, 1979).

\begin{tabular}{|c|c|c|c|c|c|c|c|c|c|c|c|}
\hline \multicolumn{4}{|c|}{ Manaus (1976) } & \multicolumn{4}{|c|}{ Porto Velho (1977) } & \multicolumn{4}{|c|}{ Itacoatiara (1977) } \\
\hline Nome vulgar & Nome científico & $\%$ & $\mathrm{t}$ & Nome vulgat & Nome científico & $\%$ & $\mathbf{t}$ & Nome vulgar & Nome científico & $\%$ & $t$ \\
\hline Tambaqui & Colossoma macropomum & 44 & 13.607 & Jatuarana & Brycon sp. & 23 & 136 & Jaraqui & Semaprochilodus sp. & 62 & 2.903 \\
\hline Jaraqui & Semaprochilodus sp. & 16 & 4.925 & Curimatã & Prochilodus nigricans & 19 & 113 & Aruanã & Osteoglossum & 6 & 268 \\
\hline Curimatã & Prochilodus nigricans & 11 & 3.444 & Pacu & Mytossoma sp. & 13 & 76 & & & & \\
\hline Pacu & Mylossoma sp. & 5 & 1.625 & Dourado & $\begin{array}{l}\text { Brachyplatystoma } \\
\text { flavicans }\end{array}$ & 11 & 65 & $\begin{array}{l}\text { Cuiú-cuiú } \\
\text { Acari }\end{array}$ & $\begin{array}{l}\text { Oxydoras niger } \\
\text { Pterygoplichthys sp. }\end{array}$ & $\begin{array}{l}5 \\
4\end{array}$ & $\begin{array}{l}251 \\
175\end{array}$ \\
\hline Aracu & Schizodon sp. & 3 & 1.010 & Tambaqui & Colossoma macropomum & 10 & 58 & Dourado & $\begin{array}{l}\text { Brachyplatystoma } \\
\text { flavicans }\end{array}$ & 3 & 120 \\
\hline & $\begin{array}{l}\text { Leporinus sp. } \\
\text { Rhithydon sp. }\end{array}$ & 4 & 1.111 & $\begin{array}{l}\text { Jaraqui } \\
\text { Branquinha }\end{array}$ & $\begin{array}{l}\text { Semaprochilodus sp. } \\
\text { Casterotomus sp. }\end{array}$ & 7 & 45 & Tambaqui & Colossoma macropomum & 2 & 91 \\
\hline Matrinchã & Brycon sp. & 3 & 1.047 & & $\begin{array}{l}\text { Potamorhina sp. } \\
\text { Semitapicis sp. }\end{array}$ & 4 & 26 & Tucunaré & Cichla ocellaris & 2 & 89 \\
\hline Tucunaré & Cichla ocellaris & 3 & 914 & & & & & Pirarucu & Arapaima gigas & 2 & 76 \\
\hline Sardinha & Triportheus sp. & 2 & 704 & $\begin{array}{l}\text { Piraiba } \\
\text { filhote }\end{array}$ & $\begin{array}{l}\text { Brachyplatystoma } \\
\text { filamentosum }\end{array}$ & 4 & 22 & Cará-açu & Astronotus ocellatus & 2 & 71 \\
\hline Branquinha & $\begin{array}{l}\text { Castêrótomus sp. } \\
\text { Potamorphina } \mathrm{sp} \text {. }\end{array}$ & 2 & 539 & Tucunaré & Cichia ocellaris & 3 & 17 & Surubim & $\begin{array}{l}\text { Pseudoplatystoma } \\
\text { fasciatum }\end{array}$ & 2 & 56 \\
\hline & Semitapicis sp. & & & Pirapitinga & Colossoma brachypomum & 2 & 11 & & & & \\
\hline
\end{tabular}


nias com peixes só pode ser realizado com grandes despesas, a piscicultura seria uma possibilidade de garantir a produção local de proteína (Saint-Paul \& Werder, 1977).

Primeiros experimentos de alimentação de peixes regionais foram feitos com Colossoma macropomum, Brycon sp., Mylossoma sp. e Semaprochilodus sp. (Saint-P.aul \& Werder, 1977, Werder \& Saint-Paul, 1978). Todas as espécies mostraram boas condições para a piscicultura, tanto na monocultura (Colossoma macropomum, Brycon sp., Mylossoma sp.) como na policultura (as espécies mencionadas, mais o Semaprochilodus). Com o Colossoma macropomum já foram feitos experimentos de alimentação nas estaçōes de piscicultura de DNOCS, Ceará (Jeffrey, 1972; Bezerra da Silva, 1975; Lovshin. 1974; Lovshin et al., 1974) e na Venezuela (Bermudez et al., 1979) .

Com estes dados, já disponiveis, uma forma de piscicultura de nível técnico baixo poderia ser introduzida, utilizando detritos da agricultura como p.ex. as frutas de seringa (Hevea brasiliensis ou Hevea spruceana). Esta forma de piscicultura foi desenvolvida na Ásia e já praticada, há alguns séculos, com muito sucesso. (Tapiador et al., 1977) .

\section{CONCLUSÃO}

A Amazônia dispõe de vários biótopos aọuáticos de grande importância na alimentação e reprodução dos peixes. A vegetação flutuante p.ex., tem importância como grande viveiro de alevinos e como biótopo de uma grande quantidade de peixes comerciais adultos. O igapó representa, com os frutos e sementes, uma grande oferta de alimentação para os peixes.

Para poder abastecer a população regularmente com proteína animal de pescado, será necessário tomar medidas que garantam o aproveitamento máximo dos estoques pesqueiros explorados e não explorados.

Isso não significa apenas um controle do material de pesca, introdução de uma fase de proteção, melhoramento da tecnologia de pescado e aproveitamento de outras espécies de peixes, mas principalmente, uma proteção dos biótopos dos peixes. Isso inclui: evitar qualquer poluição na água e preservar os biótopos da várzea e do igapó, como áreas mais importantes para o crescimento da maioria des peixes comerciais da região amazônica.

\section{BIBLIOGRAFIA}

BAyi.eY, P. B.

1978 - Avaliação dos estoques pesqueiros : Avaliação inicial da pesca. Acta Amazoníca, (no prelo).

Bermudez, D. A.; Prada C., N. R. \& Kossowski, C.

1979 - Ensayo sobre la reproduccion de Cachama Colossoma Macropomum (Courvier) 1818 en cautiverio. Universidad Centro Occidental, Escuela de Agrononomia. 23p.

BIZZERRA DA SILVA, A.

1975 - From research institutions : Induced breeding of tambaqui and pirapitinga. FAO Aqua culture Bulletin, 7 (1/2): $5 p$.

CODEAMA

1977 - Consumo alimentício em quantidades e outros indicadores econômicos 1. 1.: Pesquisa sobre Orçamentos Familiares, Governo do Estado do Amazonas, Manaus, Brasil. 174p.

GeIsLER, R.; KNöPPEL, H. A. \& SIOLI, H.

1971 - Okologie der Süsswasserfische Amazoniens. Stand und Zukunftsaufgaben der Forschung. Naturwiss., 58: 303-311.

Goulding, M.

1979 - Ecologia da pesca no rio Madeira. Manaus, INPA. 172p.

HAFFER, $\mathrm{H}$.

1974 - Avian speciation in Tropical South America. Publ. Nuttal. Ornith. Club, N. 14 , Cam. bridge, Massachusetts. 390p.

Honda, E. M. S.; Correa, C. M.; Castelo, F. P. \& Zapelini, E. A.

1975 - Aspectos gerais do pescado no Amazonas. Acta Amazonica, 5 (1): 87-94.

JEFFREY, N. B.

1972 - Progress report on fisheries development in northeastern Brazil. I. Aquaculture. Auburn Univ. (Ala.) Agr. Exp. Sta, ICA R\&D Series, 1: 3-10.

JUNK, W. J.

1970 - Investigations on the ecology and productionbiology of the "floating meadows" (PaspaloEchinochoetum) on the Middle Amazon. Part 1: The floating vegetation an its ecology. Amazoniana, 2 (4): 449-495.

1973a - Investigations on the ecology and production-biology of the "floating meadows" (Paspalo-Echinochloetum) on the Middle Amazon. Part II: The aquatic fauna in the root zone of floating vegetation. Amazoniana, 4 (1): 9-102. 
1973b- Faunistisch-ökologische Untersuchungen als Möglichkeit der Definition von Lebensräumen, dargestellt an Überschwemmungsgebieten. Amazoniana, 4 (3): 263-272.

1975 - Aquatic Wildlife and Fisheries. The Use of Ecological Guidelines for Development in the American Humid Tropics (IUCN), Caracas, Venezuela, 1974. p. 109-125.

Junk, W. J. \& HoNDA, E. M. S.

1976 - A pesca na Amazônia. Aspectos ecológicos e econômicos. Anais do $10^{\circ}$ encontro nacional sobre limnologia, piscicultura e pesca continental, Belo Horizonte, 1975. p. 211-226.

LovshiN, L. L.

1974 - From research institutions: Pond culture of tambaqui; pond culture of pirapitinga. FAO Aquaculture Bulletin, 6 (2/3): 9-10.

Lovshin, L.L.; Bezerra da SIlva, A.; Fernandes, J.A. \& CARNEIRO-SOBRINHO, A.

1974 - Preliminary pond culture test of Pirapitinga (Mylossoma bidens) and Tambaqui (Colossoma bidens) from the Amazon river basin. CARPAS/6/74/SE 24.

KJRSChbaum, F.

1979 - Reproduction of the weakly electric fisn Eigenmannia virescens (Rhamphichyidae, Teleostei) in captivity. Behav. Ecol. Sociobiol., 4: 331-355.

KNÖPPEL, H. A.

1970 - Food of Central Amazonian fishes. Contribution to the nutrient-ecology of Amazonian rain-forest streams. Amazoniana, 2 (3): 257352.

MCCONNELL, R. H.

1975 - Fish communities in tropical freshwater. Longman Inc., New York. 337p.

Petrere, M. JR.

1978 - Pesca e esforço de pesca no Estado do Amazonas. II. Locais, aparelhos de captu. ra e estatísticas de desembarque. Acta Amazonica, 8 (3) Supl. 3: 5-54.
ROBERTS, T. R.

1972 - Ecology of fishes in the Amazon and Congo basins. Bull. Mus. Comp. Zool,, 143 (2): 117-147.

SiINT-Paul, U. \& Werder, U.

1977 - Aspectos generales sobre la piscicultura en el Amazonas y resultados preliminares de experimentos de alimentacion de Brycon melanopterum con raciones peletizadas con diferentes composiciones. I Simposio de la Asociacion Latinoamericana de Acuicultura, Maracay, Venezuela: $19 p$.

SCHMIDT, G. W.

1973 - Primary production of phytoplancton in che three types of Amazonian waters. II. The limnology of a tropical flood-plain lake in Central Amazonia (Lago de Castanho). Ama. zoniana, 4 (2): 139-240.

Shrimpton, R.; Giugliano, R. \& Rodrigues, N. M.

1978 - Consumo de alimentos e alguns nutrientes em Manaus, 1973-1974. Acta Amazonia, 9 (1): $117-141$

SroLI, H.

1950 -- Das Wasser im Amazonasgebiet. Forschung und Fortschritte, 21/22: 274-280.

1967 - Studies in Amazonian waters. Atas do Simp. sobre a Biota Amazônica, 3: 9-50.

SMITH, N. J. H.

1979 - A pesca no rio Amazonas. INPA, Manaus, $154 p$.

Tapiador, D.D.; Henderson, H.F.; Delmendo, M.N.

\& Tsutsui, $\mathrm{H}$.

1977 - Freshwater fisheries and aquacultura in Chi. na. FAO Fish. Techn. Paper, 168: 84p.

WELCOMME, R. L.

1976 - Some general and theoretical considerations on the fish yield of African rivers. J. Fish. Biol., 68: 351-364.

WERDER, U. \& SAINT-PaUl, U.

1978 - Feeding trials with herbivorous and omnivo. rous Amazonian fishes. Aquaculture, 15: 175-177.

WILHELMY, $\mathrm{H}$.

1970 - Amazonien als Lebens - und Wirtschafis. raum. Staden-Jahrbuch, 18: 9-31. 